\title{
Forecasting COPD hospitalization in the clinic: optimizing the chronic respiratory questionnaire
}

\author{
This article was published in the following Dove Press journal: \\ International Journal of COPD \\ 22 October 2015 \\ Number of times this article has been viewed
}

\author{
Beatriz Abascal-Bolado' \\ Paul J Novotny ${ }^{2}$ \\ Jeff A Sloan ${ }^{2}$ \\ Craig Karpman ${ }^{3}$ \\ Megan M Dulohery ${ }^{3}$ \\ Roberto P Benzo ${ }^{3}$
}

'Pulmonary Division, Instituto de Investigación Sanitaria Valdecilla (IDIVAL), Santander, Spain; ${ }^{2}$ Department of Cancer Center Statistics, Health Science Research, ${ }_{3}^{3}$ Mindful Breathing Laboratory, Division of Pulmonary and Critical Care Medicine, Mayo Clinic, Rochester, MN, USA

Correspondence: Roberto P Benzo Mindful Breathing Laboratory, Division of Pulmonary and Critical Care Medicine, Mayo Clinic, 200 First Street Southwest, Rochester, MN 55905, USA

$\mathrm{Tel}+\mathrm{I} 507284056$ I

Fax + I 5072664372

Email benzo.roberto@mayo.edu

\begin{abstract}
Purpose: Forecasting hospitalization in patients with COPD has gained significant interest in the field of COPD care. There is a need to find simple tools that can help clinicians to stratify the risk of hospitalization in these patients at the time of care. The perception of quality of life has been reported to be independently associated with hospitalizations, but questionnaires are impractical for daily clinical use. Individual questions from valid questionnaires can have robust predictive abilities, as has been suggested in previous reports, as a way to use patient-reported outcomes to forecast important events like hospitalizations in COPD. Our primary aim was to assess the predictive value of individual questions from the Chronic Respiratory Questionnaire Self-Assessment Survey (CRQ-SAS) on the risk of hospitalization and to develop a clinically relevant and simple algorithm that clinicians can use in routine practice to identify patients with an increased risk of hospitalization.
\end{abstract}

Patients and methods: A total of 493 patients with COPD prospectively recruited from an outpatient pulmonary clinic completed the CRQ-SAS, demographic information, pulmonary function testing, and clinical outcomes. The cohort had a mean age of 70 years, was $54 \%$ male, with forced expiratory volume in 1 second percentage predicted 42.8 \pm 16.7 , and modified Medical Research Council dyspnea scale score of $2 \pm 1.13$.

Results: Our analysis validated the original CRQ-SAS domains. Importantly, recursive partitioning analysis identified three CRQ-SAS items regarding fear or panic of breathlessness, dyspnea with basic activities of daily living, and depressive symptoms that were highly predictive of hospitalization. We propose a robust (area under the curve $=0.70$ ) but short and easy algorithm for daily clinical care to forecast hospitalizations in patients with COPD.

Conclusion: We identified three themes - fear of breathlessness, dyspnea with basic activities of daily living, and depressive symptoms - as important patient-reported outcomes to predict hospitalizations, and propose a short and easy algorithm to forecast hospitalizations in patients with COPD.

Keywords: quality of life, COPD, exacerbation

\section{Introduction}

COPD is one of the main causes of morbidity and mortality worldwide, and is expected to become the third-leading cause of death and the fifth-leading cause of disabilityadjusted life years lost in 2020. ${ }^{1}$ Patients with COPD suffer recurrent exacerbations that require hospitalization. Reducing admissions to the hospital is one of the primary aims in the management of the disease, ${ }^{2}$ due to the high burden to the patient and also the economic and social cost. ${ }^{3}$ Furthermore, GOLD (Global initiative for chronic Obstructive Lung Disease) recently proposed a way to categorize risk in patients with COPD using history of exacerbations for identifying patients that have a higher risk of being hospitalized. There is a need for simple ways to identify patients who are at the highest risk of being hospitalized within the clinic, in order to design preventive interventions and proper care, including palliative care. 
Quality of life (QoL), measured with disease-specific questionnaires, has been shown to offer additional information to predict COPD patients' risk of hospitalization. ${ }^{4}$ However, disease-specific questionnaires are currently done in the context of clinical trials, and are rarely used in daily practice. Not using the predictive information from such questionnaires as the Chronic Respiratory Questionnaire Self-Administered Survey (CRQ-SAS) $)^{5-7}$ or St George's Respiratory Questionnaire (SGRQ) ${ }^{8}$ may be a "missing opportunity" for better forecasting the risk of hospitalization. While the entire questionnaire might not be feasible for routine use, some questions might be significantly associated with the likelihood of hospitalization and merit further investigation. For example, while inflammatory and physiologic changes associated with COPD have been implicated in the onset of depression and anxiety, ${ }^{9}$ the perception of QoL is a better predictor of difficult emotions in COPD than biological or physiological markers. ${ }^{10}$ The objective of the present study was to investigate the predictive value of individual questions from a disease-specific QoL questionnaire, the CRQ-SAS, on the risk of hospitalization in a well-characterized cohort of COPD patients. The ultimate goal of this work is to develop a clinically relevant and easy algorithm that clinicians can use in routine practice to identify patients with an increased risk of hospitalization.

\section{Patients and methods}

Patients with COPD $(n=493)$ were prospectively recruited from an outpatient pulmonary clinic at Mayo Clinic in Rochester, MN, USA. The following inclusion criteria were used in an attempt to obtain a uniform patient population: diagnosis of COPD based on the GOLD 2011 guidelines, ${ }^{11}$ age $>40$ years, history of smoking more than 10 packyears, and the ability to complete questionnaires. This study received Mayo Clinic Institutional Review Board approval, and all patients signed research consent.

At the time of recruitment, investigators recorded demographics, clinical information, and health care utilization (hospitalization and ER visits) during the previous year and self-reported by participants. Pulmonary function tests were performed according to the current guidelines and established reference values. ${ }^{12}$ Dyspnea status was assessed using the modified Medical Research Council (mMRC) dyspnea scale (range: 0-4), as has been previously described. ${ }^{13}$ Perceived QoL was assessed using the CRQ-SAS, a 20-item validated questionnaire that measures the health status of patients with COPD. The inventory is divided into four dimensions: dyspnea, fatigue, emotional function, and mastery. Lower scores in each dimension indicate a greater degree of dysfunction on a 7-point scale. ${ }^{67}$ Finally, patients were classified subsequently according to the GOLD severity and risk criteria (GOLD 2013) into GOLD A, GOLD B, GOLD C, and GOLD D groups. ${ }^{14}$ The composite index of COPD severity (age, dyspnea, obstruction) ${ }^{15}$ was calculated for all patients.

\section{Statistical analysis}

The goal of this study was to define subsets of items (individual questions from the CRQ-SAS) that could predict the risk of hospitalization after adjusting for age, lung function, history of hospitalizations, and mMRC dyspnea that can be used in daily clinical practice to make decisions on individuals at highest risk of hospitalization. Methods used to define the subsets included variable cluster analysis, factor analysis using scree plots and varimax rotation, recursive partitioning (classification and regression tree [CART] models), univariate and stepwise logistic regression models, and model validation using bootstrapping. Recursive partitioning was done using RPART version 4.1-8. ${ }^{16}$ All other analyses were done using SAS 9.2 (SAS Institute Inc, Cary, NC, USA).

Development of the algorithm to identify patients at risk of hospitalization began with a confirmatory analysis of the internal psychometric structure of the existing measures. Factor- and cluster-analysis routines were followed to demonstrate that indeed the internal structure of the original measures was demonstrated in our application setting.

Univariate logistic models were fitted to see how well each of the individual questions and subscales could predict hospitalizations. Collinearity diagnostics were then run to determine whether any combinations of variables were too highly correlated to be included together in multivariate modeling. These diagnostic measures showed that the four CRQ subscales (mastery, dyspnea, fatigue, and emotional) were highly related to the individual CRQ questions, so subsequent multivariate models only used individual CRQ items. No other multicollinearity concerns were found. The logistic regression models then identified a subset of the individual items that were related to the incidence of hospitalization, indicating that a small number of items might be useful in identifying patient subgroups at higher risk of hospitalization. The Akaike information criterion was used as the primary guide for item selection, along with the $c$-statistic.

CART models were used to find combinations of items that could predict hospitalization using the variables identified in the previous factor analysis, cluster analysis, and logistic regression work. CART models were created once using only the individual CRQ questions and once using the 
CRQ variables plus sex, age, forced expiratory volume in 1 second $\left(\mathrm{FEV}_{1}\right)$ percentage predicted, and mMRC scale. Stepwise logistic regression models were then used to confirm and refine the predictive power of the CART models. Finally, the stepwise logistic models were confirmed by applying stepwise logistic modeling to 1,000 bootstrapped samples.

\section{Results}

Demographics and baseline characteristics are shown in Table 1. Cluster and factor analysis arrived at the same conclusions and verified the original CRQ-SAS domains. These two multivariate methods resulted in the following four groups of the CRQ-SAS variables: group 1, questions $1-5$; group 2, questions $8,11,15$, and 17; group 3 , questions $6,12,14,16,18$, and 20; and group 4, questions 7, 9, 10, 13 , and 19. These four factors are the same as the four factors defined in the development of the CRQ: dyspnea, fatigue, emotional, and mastery. ${ }^{5}$ The only exception was that question 9 clustered with the mastery variables in this analysis, even though it was originally part of the emotional subscale. This is not a surprising result, since question 9 contains both emotional issues and concerns about how well the subjects can overcome COPD barriers.

Logistic regression models were used to determine variables associated with hospitalization. Before running these models, collinearity diagnostics were checked. The subscales based on factor analysis were strongly related to the individual CRQ-SAS variables, so those variables were

Table I Baseline characteristics

\begin{tabular}{ll}
\hline Variable & Mean \pm SD/n (\%) \\
\hline $\mathrm{n}$ & 493 \\
Age (years) & $70 \pm 9.5$ \\
Sex & \\
$\quad$ Male & $267(54.4)$ \\
Active smokers (\%) & 13 \\
BMI & $28.6 \pm 6.5$ \\
PFT data & \\
FEV $\%$ predicted & $42.8 \pm 16.7$ \\
mMRC dyspnea scale & $2 \pm 1.13$ \\
Exacerbations $\geq 2$ previous year & $169(42)$ \\
Hospital admission previous year & $163(33)$ \\
ADO index (range: $0-14)$ & $8.8 \pm 2.1$ \\
GOLD risk groups & \\
A & $89(18.0)$ \\
B & $86(17.4)$ \\
C & $66(13.3)$ \\
D & $252(51.1)$ \\
\hline
\end{tabular}

Abbreviations: SD, standard deviation; BMI, body mass index; PFT, pulmonary function test; $F E V_{1}$, forced expiratory volume in I second; mMRC, modified Medical Research Council; ADO, age, dyspnea, obstruction; GOLD, Global initiative for chronic Obstructive Lung Disease. not used together in the logistic models. All of the other variables had condition numbers less than 100 , indicating there were no other collinearity concerns.

Recursive partitioning was done using just the CRQ-SAS variables and again using the CRQ-SAS variables along with the mMRC dyspnea scale, age, sex, and $\mathrm{FEV}_{1} \%$ predicted. The resulting CART model using the CRQ-SAS variables demonstrated an algorithm to predict hospitalization (Figure 1). Univariate logistic modeling (Table 2) showed that $\mathrm{FEV}_{1} \%$ predicted, mMRC dyspnea scale, each of the individual CRQ-SAS questions, the subscales created from factor analysis, and the CART groups were all significantly related to hospitalization. Age, sex, and active smoking were not associated with hospitalization rates. The two different CART models (CRQ-SAS variables alone versus CRQ-SAS variables plus other variables: mMRC dyspnea scale, $\mathrm{FEV}_{1} \%$ predicted, age, and sex) performed almost the same in predicting the risk of hospitalizations. In fact, the CART model using just the CRQ-SAS variables had a better $c$-statistic (0.70) than the other CART model using the mMRC dyspnea scale, $\mathrm{FEV}_{1} \%$ predicted, age, and sex $(c=0.684)$. Stepwise logistic modeling found that the CART model using the CRQ-SAS variables was the best predictor of the risk of hospitalization (Table 3).

The algorithm obtained through the CART modeling (Figure 1) identified two subsets of patients that accounted for $40 \%$ of the sample with a combined hospitalization risk of $50 \%$ (double the risk of hospitalization compared to the rest of the patients). The first subset, approximately $20 \%$ of the sample (103 of 493), indicated that they felt "fear or panic" when they were short of breath most of the time or all the time. These participants carried a $57 \%$ risk of hospitalization compared to only $27 \%$ for the rest of the sample (all other groups combined). The second subset, approximately a further $20 \%$ of the sample (101 of 493), included individuals who indicated they felt at least mildly short of breath while caring for their basic needs and also "felt discouraged or down in the dumps", at least a little of the time, and had a $43 \%$ risk of hospitalization.

\section{Discussion}

We built an algorithm using three questions from the CRQSAS following a rigorous statistical method that can help clinicians to forecast risk of hospitalization and increase our portfolio of methods to determine hospitalization risk, an event associated with poor prognosis and mortality. ${ }^{17}$

We identified important outcomes in COPD - fear of breathlessness, dyspnea with activities of daily living, and 


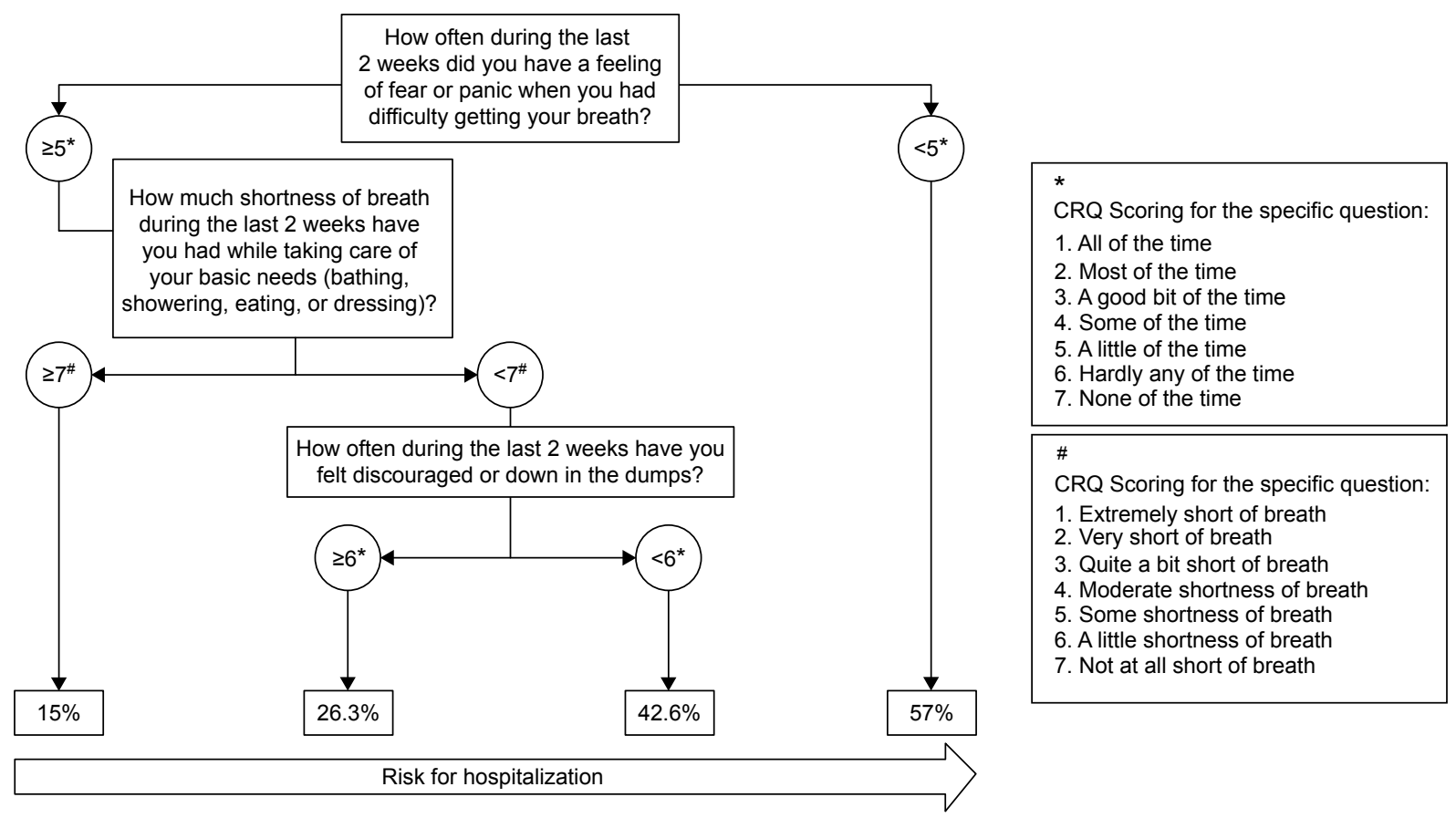

Figure I Algorithm proposed to predict hospitalization in patients with COPD.

depressive symptoms (Figure 1) - that have been previously reported with an increased risk of hospitalization. ${ }^{14,18-20}$ Importantly, we defined specific cut points (a defined score for each question) that made them simple for interpretation and definition of risk.

We identified a simple way to test the construct of fear of breathlessness in COPD that is associated with hospitalizations. The construct of fear has been shown to have an impact on disease disability in COPD. ${ }^{21}$ Specific measures of the construct of fear to use in daily care are lacking, but currently existing measures have been reported to be predictive of COPD-treatment outcomes after controlling for general anxiety. ${ }^{22}$ Disease-specific fear is defined as anxiety in the face of severe physical symptoms and their consequences. ${ }^{23}$ Keil et al showed in 2011 the importance of specific fears in the context of COPD and their association with disability. ${ }^{21}$ Our study supports the hypothesis of Keil et al feeling fear or panic due to shortness of breath was the most predictive question in our algorithm: a 57\% risk of hospitalization.

Our results, which further emphasize the importance of symptoms of depression and anxiety (fear), have profound consequences for the health of patients with COPD. Anxiety (fear) is associated with increased risk of exacerbations, poorer health-related QoL, worse physical activity ${ }^{19}$ relapse within 1 month of receiving emergency treatment, ${ }^{24}$ and hospital readmission. ${ }^{25}$ Depression is associated with increased mortality, impaired health-related QoL, and excessive health-care-utilization rates and costs, including longer hospital stay after acute exacerbation, ${ }^{20}$ increased risk of exacerbation and hospital admission, ${ }^{26}$ hospital readmission, ${ }^{27}$ and lower physical activity. ${ }^{28}$

We support and extend previous reports about the impact emotions (anxiety and depression) have on health care utilization (in addition to physical functioning and QoL). ${ }^{19,29,30}$ GOLD has recently highlighted the importance of effective screening and management of anxiety and depression. ${ }^{14}$ Furthermore, gathering patient-reported outcome data regarding sensitive issues, such as fear and depression, from a questionnaire rather than through a personal and potentially difficult conversation has been seen to elicit information that a patient may not be willing to verbalize to a health care provider. ${ }^{31,32}$

Our findings also extend previous reports suggesting that individual items within questionnaires can be as sensitive, if not more sensitive, than the overall scores and content of larger questionnaires to forecast specific outcomes. ${ }^{33,34}$ Furthermore, items drawn from general questionnaires have been demonstrated to be useful indicators for more refined clinical interventions. ${ }^{35}$ The overall measures and longer questionnaires remain useful tools, but by looking at the psychometrics of individual items we can capitalize on the most clinically relevant information.

We followed a strict psychometric approach with multiple sensitivity analyses via alternative analytical approaches 
Table 2 Univariate logistic models predicting hospitalizations

\begin{tabular}{|c|c|c|c|c|}
\hline Variable & $P$-value & Odds ratio $(95 \% \mathrm{Cl})$ & c-statistic & AIC \\
\hline Male & 0.278 & $1.23(0.845-1.795)$ & 0.53 & 626 \\
\hline Age & 0.638 & $0.99(0.976-1.015)$ & 0.51 & 630 \\
\hline $\mathrm{FEV}, \%$ predicted & 0.003 & $0.98(0.97 I-0.994)$ & 0.58 & 608 \\
\hline $\mathrm{mMRC}$ & $<0.0001$ & I.7I (I.42I-2.076) & 0.65 & 595 \\
\hline QI: SOB while angry or upset & 0.0002 & $0.79(0.697-0.894)$ & 0.62 & 525 \\
\hline Q2: SOB caring for basic needs & $<0.000 \mathrm{I}$ & $0.75(0.667-0.849)$ & 0.63 & 595 \\
\hline Q3: SOB walking & $<0.0001$ & $0.74(0.656-0.839)$ & 0.64 & 586 \\
\hline Q4: SOB performing chores & $<0.0001$ & $0.72(0.64 I-0.820)$ & 0.65 & 568 \\
\hline Q5: SOB in social activities & $<0.000$ I & $0.73(0.643-0.833)$ & 0.64 & 536 \\
\hline Q6: felt frustrated or impatient & 0.002 & $0.80(0.694-0.923)$ & 0.59 & 607 \\
\hline Q7: feeling fear or panic with SOB & $<0.0001$ & $0.63(0.553-0.728)$ & 0.67 & 573 \\
\hline Q8: fatigue & $<0.0001$ & $0.76(0.670-0.87 I)$ & 0.61 & 609 \\
\hline Q9: embarrassed by cough or heavy breathing & 0.001 & $0.82(0.737-0.933)$ & 0.59 & 616 \\
\hline QI0: confident could deal with illness & $<0.0001$ & $0.76(0.678-0.868)$ & 0.62 & 608 \\
\hline QII: energy & $<0.0001$ & $0.70(0.591-0.835)$ & 0.62 & 607 \\
\hline Q12: feel upset, worried, or depressed & 0.0002 & $0.76(0.666-0.883)$ & 0.60 & 600 \\
\hline QI3: felt in control of breathing problems & $<0.0001$ & $0.80(0.723-0.892)$ & 0.61 & 604 \\
\hline QI4: felt relaxed & 0.013 & $0.85(0.75 \mathrm{I}-0.968)$ & 0.57 & 615 \\
\hline Q15: felt low in energy & 0.0001 & $0.75(0.657-0.87 I)$ & 0.61 & 606 \\
\hline Q16: felt discouraged & $<0.0001$ & $0.75(0.655-0.866)$ & 0.61 & 608 \\
\hline Q17: felt worn out or sluggish & 0.0004 & $0.78(0.682-0.897)$ & 0.60 & 605 \\
\hline Q18: happy with personal life & 0.0003 & $0.77(0.669-0.887)$ & 0.60 & 608 \\
\hline Q19: felt upset or scared when difficult to get breath & $<0.000$ I & $0.65(0.569-0.745)$ & 0.67 & 574 \\
\hline Q20: felt restless, tense, or uptight & 0.0001 & $0.76(0.665-0.877)$ & 0.60 & 613 \\
\hline CART groups using all variables & $<0.0001$ & $\mathrm{I} .77(1.504-2.094)$ & 0.68 & 548 \\
\hline CART groups using only CRQ-SAS & $<0.0001$ & $1.64(1.434-1.885)$ & 0.70 & 556 \\
\hline Factor analysis: factor I & $<0.0001$ & $0.72(0.619-0.852)$ & 0.61 & 615 \\
\hline Factor analysis: factor 2 & $<0.0001$ & $0.67(0.586-0.781)$ & 0.65 & 590 \\
\hline Factor analysis: factor 3 & $<0.0001$ & $0.70(0.605-0.832)$ & 0.62 & 612 \\
\hline Factor analysis: factor 4 & $<0.0001$ & $0.64(0.550-0.745)$ & 0.66 & 595 \\
\hline Cluster analysis: cluster I & $<0.0001$ & $0.72(0.619-0.852)$ & 0.61 & 615 \\
\hline Cluster analysis: cluster 2 & $<0.0001$ & $0.64(0.550-0.745)$ & 0.66 & 590 \\
\hline Cluster analysis: cluster 3 & $<0.0001$ & $0.70(0.605-0.832)$ & 0.62 & 612 \\
\hline Cluster analysis: cluster 4 & $<0.0001$ & $0.67(0.586-0.78 I)$ & 0.65 & 595 \\
\hline
\end{tabular}

Abbreviations: $\mathrm{Cl}$, confidence interval; AIC, Akaike information criterion; FEV , forced expiratory volume in I second; mMRC, modified Medical Research Council; SOB, shortness of breath; CART, classification and regression tree; CRQ-SAS, Chronic Respiratory Questionnaire Self-Assessment Survey.

and bootstrapping resampling sets of 1,000 patients. Further application of our algorithm is needed to validate its clinical utility and generalizability. The algorithm is intended to be a supplementary source of information that the clinician can incorporate into their personal assessment of the patient that can impact the monitoring and care plan for the patient.

Table 3 Stepwise logistic model results

\begin{tabular}{lll}
\hline Model & c-statistic & AIC \\
\hline CART model with only CRQ-SAS variables & 0.70 & 561.522 \\
CART model with all variables* & 0.68 & 551.709 \\
\hline
\end{tabular}

Note: *CRQ-SAS variables, mMRC dyspnea scale, age, sex, and FEV \% predicted. Abbreviations: AIC, Akaike information criterion; CART, classification and regression tree; CRQ-SAS, Chronic Respiratory Questionnaire Self-Assessment Survey; mMRC, modified Medical Research Council; FEV , forced expiratory volume in I second.
Importantly, the algorithm incorporates patient-reported information that has not been used routinely or systematically in clinical practice. Current algorithms in the area of COPD care have prognostic characteristics that are similar to what we have produced. The body mass, obstruction, dyspnea, and exercise capacity index that has universal application in clinical practice have an area under the curve of 0.74 , which is comparable to the area under the curve of 0.70 obtained for our algorithm. ${ }^{36}$

Our group previously showed that a single item measuring QoL was actually more informative and sensitive to change than longer scales purported to measure the same thing. ${ }^{37}$ Specifically, in COPD we initially reported that a single general question on health status is independently associated with the risk of hospitalization. ${ }^{18}$ 


\section{Limitations}

The retrospective health-care-utilization measure (history of a hospitalization in the last year) can be viewed as a limitation. However, a recent report from the ECLIPSE group ${ }^{38}$ has clearly shown a very strong association between a hospitalization in the previous year and hospitalization in the subsequent year, supporting the validity of the measure used in this study.

There are (undoubtedly) more variables involved to accurately predict individualized risk for hospitalization (a previous hospitalization and GOLD risk assessment); our results provide a simple screening tool to identify a subset of the population that might benefit from closer monitoring, specific therapies, or even palliative care. In particular, the data indicate that if a patient perceives fear related to breathlessness, their risk of hospitalization doubles from a mean value of $30 \%$ in the COPD-severe population to approximately $50 \%{ }^{39}$ This allows the limited resources available to the clinical team to be directed to patients who are more likely to be hospitalized.

This algorithm to identify patients at higher risk of hospitalization will have false positives and negatives; however, it is not intended as a diagnostic laboratory test, but a supplementary source of information for the clinician.

\section{Conclusion}

We created a simple algorithm following a very robust statistical method that may become informative to the practicing clinician to forecast the risk of hospitalization in COPD patients. We identified clinically relevant constructs (fear of breathlessness, significant dyspnea with activities of daily living, and depressive symptoms) embedded in the CRQ questionnaire that are independently associated with hospitalization risk. Our work is not implying that we have a better way to predict hospitalization, but an alternative way to augment our accuracy in forecasting the risk of hospitalization and the opportunity to provide our patients the most individualized and precise care possible.

\section{Acknowledgment}

This research was supported by grant 1R01HL094680 from the National Institutes of Health (NIH), Roberto Benzo PI.

\section{Disclosure}

The authors report no conflicts of interest in this work.

\section{References}

1. Murray CJ, Lopez AD. Alternative projections of mortality and disability by cause 1990-2020: Global Burden of Disease Study. Lancet. 1997;349(9064):1498-1504.

2. Vestbo J, Hurd SS, Agusti AG, et al. Global strategy for the diagnosis, management, and prevention of chronic obstructive pulmonary disease: GOLD executive summary. Am J Respir Crit Care Med. 2013;187(4):347-365.

3. Sullivan SD, Ramsey SD, Lee TA. The economic burden of COPD. Chest. 2000;117(2 Suppl):5S-9S.

4. Fan VS, Curtis JR, Tu SP, McDonell MB, Fihn SD. Using quality of life to predict hospitalization and mortality in patients with obstructive lung diseases. Chest. 2002;122(2):429-436.

5. Guyatt GH, Berman LB, Townsend M, Pugsley SO, Chambers LW. A measure of quality of life for clinical trials in chronic lung disease. Thorax. 1987;42(10):773-778.

6. Schünemann HJ, Goldstein R, Mador MJ, et al. A randomised trial to evaluate the self-administered standardised chronic respiratory questionnaire. Eur Respir J. 2005;25(1):31-40.

7. Schünemann HJ, Griffith L, Jaeschke R, et al. A comparison of the original chronic respiratory questionnaire with a standardized version. Chest. 2003;124(4):1421-1429.

8. Jones PW, Quirk FH, Baveystock CM, Littlejohns P. A self-complete measure of health status for chronic airflow limitation. The St George's Respiratory Questionnaire. Am Rev Respir Dis. 1992;145(6): 1321-1327.

9. Barnes PJ, Celli BR. Systemic manifestations and comorbidities of COPD. Eur Respir J. 2009;33(5):1165-1185.

10. Hanania NA, Mullerova H, Locantore NW, et al. Determinants of depression in the ECLIPSE chronic obstructive pulmonary disease cohort. Am J Respir Crit Care Med. 2011;183(5):604-611.

11. Vestbo J, Hurd SS, Rodriguez-Roisin R. The 2011 revision of the global strategy for the diagnosis, management and prevention of COPD (GOLD) - why and what? Clin Respir J. 2012;6(4):208-214.

12. Miller MR, Hankinson J, Brusasco V, et al. Standardisation of spirometry. Eur Respir J. 2005;26(2):319-338.

13. Bestall JC, Paul EA, Garrod R, Garnham R, Jones PW, Wedzicha JA. Usefulness of the Medical Research Council (MRC) dyspnoea scale as a measure of disability in patients with chronic obstructive pulmonary disease. Thorax. 1999;54(7):581-586.

14. GOLD (Global initiative for chronic Obstructive Lung Disease). Global Strategy for the Diagnosis, Management, and Prevention of Chronic Obstructive Pulmonary Disease. Bethesda (MD): GOLD; 2014.

15. Puhan MA, Hansel NN, Sobradillo P, et al. Large-scale international validation of the ADO index in subjects with COPD: an individual subject data analysis of 10 cohorts. BMJ Open. 2012;2(6):e002152.

16. Therneau TM, Atkinson EJ. An introduction to recursive partitioning using the RPART routines. 1997. Available from: http://www.mayo. edu/research/documents/biostat-61pdf/doc-10026699?_ga=1.1501622 94.1387729695.1437431825. Accessed July 21, 2015.

17. Soler-Cataluña JJ, Martínez-García MA, Román Sánchez P, Salcedo E, Navarro M, Ochando R. Severe acute exacerbations and mortality in patients with chronic obstructive pulmonary disease. Thorax. 2005;60(11): 925-931.

18. Benzo RP, Chang CC, Farrell MH, et al. Physical activity, health status and risk of hospitalization in patients with severe chronic obstructive pulmonary disease. Respiration. 2010;80(1):10-18.

19. Eisner MD, Blanc PD, Yelin EH, et al. Influence of anxiety on health outcomes in COPD. Thorax. 2010;65(3):229-234.

20. Ng TP, Niti M, Tan WC, Cao Z, Ong KC, Eng P. Depressive symptoms and chronic obstructive pulmonary disease: effect on mortality, hospital readmission, symptom burden, functional status, and quality of life. Arch Intern Med. 2007;167(1):60-67.

21. Keil DC, Stenzel NM, Kühl K, et al. The impact of chronic obstructive pulmonary disease-related fears on disease-specific disability. Chron Respir Dis. 2014;11(1):31-40. 
22. Janssens T, De Peuter S, Stans L, et al. Dyspnea perception in COPD: association between anxiety, dyspnea-related fear, and dyspnea in a pulmonary rehabilitation program. Chest. 2011;140(3):618-625.

23. Kühl K, Kuhn C, Kenn K, Rief W. [The COPD-Anxiety-Questionnaire (CAF): a new instrument to assess illness specific anxiety in COPD patients]. Psychother Psychosom Med Psychol. 2011;61(1):e1-e9.

24. Dahlén I, Janson C. Anxiety and depression are related to the outcome of emergency treatment in patients with obstructive pulmonary disease. Chest. 2002;122(5):1633-1637.

25. Gudmundsson G, Gislason T, Janson C, et al. Risk factors for rehospitalisation in COPD: role of health status, anxiety and depression. Eur Respir J. 2005;26(3):414-419.

26. Xu W, Collet JP, Shapiro S, et al. Independent effect of depression and anxiety on chronic obstructive pulmonary disease exacerbations and hospitalizations. Am J Respir Crit Care Med. 2008;178(9):913-920.

27. Coventry PA, Gemmell I, Todd CJ. Psychosocial risk factors for hospital readmission in COPD patients on early discharge services: a cohort study. BMC Pulm Med. 2011;11:49.

28. Spruit MA, Watkins ML, Edwards LD, et al. Determinants of poor 6-min walking distance in patients with COPD: the ECLIPSE cohort. Respir Med. 2010;104(6):849-857.

29. Cully JA, Graham DP, Stanley MA, et al. Quality of life in patients with chronic obstructive pulmonary disease and comorbid anxiety or depression. Psychosomatics. 2006;47(4):312-319.

30. Yohannes AM, Baldwin RC, Connolly MJ. Depression and anxiety in elderly outpatients with chronic obstructive pulmonary disease: prevalence, and validation of the BASDEC screening questionnaire. Int J Geriatr Psychiatry. 2000;15(12):1090-1096.

31. Arora NK, Jensen RE, Sulayman N, Hamilton AS, Potosky AL. Patientphysician communication about health-related quality-of-life problems: are non-Hodgkin lymphoma survivors willing to talk? JClin Oncol. 2013; 31(31):3964-3970.
32. Eremenco S, Webster K, Cella D. Quality of life evaluation in chronic illness across cultures. In: Eguchi K, Klastersky J, Feld R, editors. Current Perspectives and Future Directions in Palliative Medicine. Tokyo: Springer-Verlag; 1998:171-175.

33. Bernhard J, Sullivan M, Hürny C, Coates AS, Rudenstam CM. Clinical relevance of single item quality of life indicators in cancer clinical trials. Br J Cancer. 2001;84(9):1156-1165.

34. Sloan JA, Cella D, Frost M, Guyatt GH, Sprangers M, Symonds T. Assessing clinical significance in measuring oncology patient quality of life: introduction to the symposium, content overview, and definition of terms. Mayo Clin Proc. 2002;77(4):367-370.

35. Stucky CC, Pockaj BA, Novotny PJ, et al. Long-term follow-up and individual item analysis of quality of life assessments related to laparoscopic-assisted colectomy in the COST trial 93-46-53 (INT 0146). Ann Surg Oncol. 2011;18(9):2422-2431.

36. Celli BR, Cote CG, Marin JM, et al. The body-mass index, airflow obstruction, dyspnea, and exercise capacity index in chronic obstructive pulmonary disease. $N$ Engl J Med. 2004;350(10):1005-1012.

37. Sloan JA, Zhao X, Novotny PJ, et al. Relationship between deficits in overall quality of life and non-small-cell lung cancer survival. J Clin Oncol. 2012;30(13):1498-1504.

38. Hurst JR, Vestbo J, Anzueto A, et al. Susceptibility to exacerbation in chronic obstructive pulmonary disease. N Engl J Med. 2010;363(12): 1128-1138.

39. Agusti A, Edwards LD, Celli B, et al. Characteristics, stability and outcomes of the 2011 GOLD COPD groups in the ECLIPSE cohort Eur Respir J. 2013;42(3):636-646.
International Journal of COPD

\section{Publish your work in this journal}

The International Journal of COPD is an international, peer-reviewed journal of therapeutics and pharmacology focusing on concise rapid reporting of clinical studies and reviews in COPD. Special focus is given to the pathophysiological processes underlying the disease, intervention programs, patient focused education, and self management protocols.

\section{Dovepress}

This journal is indexed on PubMed Central, MedLine and CAS. The manuscript management system is completely online and includes a very quick and fair peer-review system, which is all easy to use. Visit http://www.dovepress.com/testimonials.php to read real quotes from published authors. 